\title{
Detuned Twin-Signal-Recycling for ultrahigh-precision interferometers
}

\author{
André Thüring, R. Schnabel, H. Lück, and K. Danzmann \\ Institut für Gravitationsphysik, Leibniz Universtität Hannover and Max-Planck-Institut für Gravitationsphysik \\ (Albert-Einstein Institut), Callinstraße 38, 30167 Hannover, Germany
}

Received December 7, 2006; accepted January 9, 2007;

posted January 29, 2007 (Doc. ID 77884); published March 19, 2007

\begin{abstract}
We propose a new interferometer technique for high-precision phase measurements such as those in gravitational wave detection. The technique utilizes a pair of optically coupled resonators that provide identical resonance conditions for the upper as well the lower phase modulation signal sidebands. This symmetry significantly reduces the noise spectral density in a wide frequency band compared with single-sideband recycling topologies of current and planned gravitational wave detectors. Furthermore, the application of squeezed states of light becomes less demanding. (c) 2007 Optical Society of America
\end{abstract}

OCIS codes: $120.3180,270.6570$.

Current interferometric gravitational wave (GW) detectors achieve a phase noise spectral density as low as $10^{-11} \mathrm{rad} / \sqrt{\mathrm{Hz}}$ between 100 and $1000 \mathrm{~Hz}$, which corresponds to a strain noise spectral density of $10^{-23} \sqrt{\mathrm{Hz}^{-1}}$. The basic topology of those detectors is that of a Michelson interferometer, with kilometerscale arms. However, to reach such high sensitivities, optical cavities play an essential role. Arm cavities and power recycling cavities, which are tuned to resonance for the laser carrier light, are used in the current GW detectors LIGO,${ }^{1}$ TAMA, ${ }^{2}$ and VIRGO ${ }^{3}$ to increase the circulating light powers. GEO $600^{4}$ uses carrier detuned (single-sideband) signal recycling ${ }^{5}$ (SR) that turns the interferometer into a resonant detector. In this scheme the upper (lower) signal sideband is resonantly enhanced and therefore the sensitivity improved, although the lower sideband (upper) is suppressed. A similar technique is planned to be used in Advanced LIGO. ${ }^{6}$ All the techniques mentioned improve the signal-to-quantum-noise ratio of the detector compared with a simple Michelson interferometer. Injection of squeezed states is another technique aimed at quantum noise reduction in interferometers. ${ }^{7}$ Current progress in the generation and control of squeezed states of light has shown that this technique can be applied in future detectors; see Ref. 8 and references given therein. Full compatibility of squeezed field injection and detuned SR was theoretically shown in Harms et al. ${ }^{9}$ considering homodyne detection. Here "full compatibility" refers to frequency-dependent squeezing of quantum radiation pressure noise and shot noise, providing a broadband sensitivity improvement. Quite generally, two or more low-loss, narrow-linewidth, and therefore longbaseline optical filter cavities are necessary to prepare the squeezed states in an optimum way to achieve this goal, as first proposed in Ref. 10. However, even in the purely shot-noise-limited regime, which is typically realized at sideband frequencies above $100 \mathrm{~Hz}$, a single-filter cavity still needs to be applied to prepare the squeezed states. ${ }^{11}$

In this Letter we propose a new technique for squeezed-state-enhanced interferometers, namely
Detuned Twin-Signal Recycling (TSR). This technique achieves an improved signal-to-shot-noise ratio compared with the detuned single-sideband SR technique over a wide part of the detection band, because upper and lower signal sidebands are resonantly enhanced simultaneously. ${ }^{12}$ We show that with this technique squeezed states can be used to reduce the interferometer's shot noise without the need for an additional filter cavity.

The shaded upper left-hand part of Fig. 1 shows a Michelson interferometer topology with the techniques of power recycling and SR as used in the GEO 600 detector. Such an interferometer is operated close to a dark fringe, which is a requirement for recycling techniques. To form a detuned SR cavity, mirror SRM is positioned such that not carrier laser light but signal light is resonating. The lower lefthand part of Fig. 1 shows the extension to employ squeezed states of light. A source of a broadband squeezed field (SQZ), a long-baseline filter cavity, and a Faraday rotator for coupling to the signal modes is
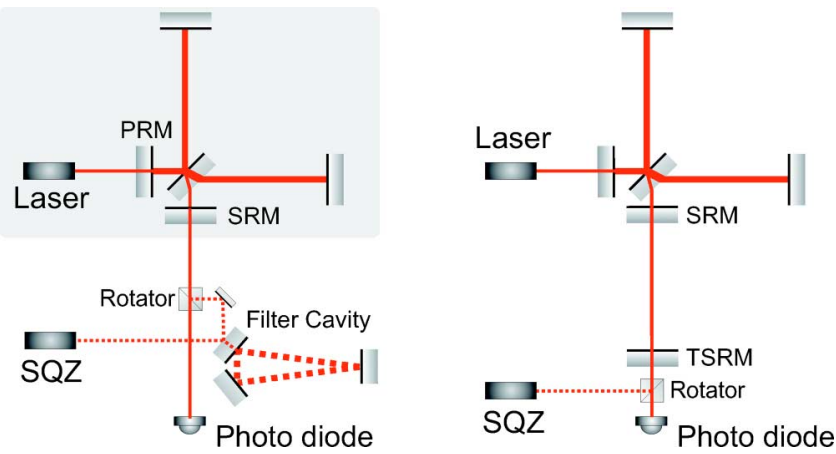

Fig. 1. (Color online) Top left (shaded), topology of the current GW detector GEO 600. The mirror PRM in the laser input port realizes so-called power recycling. The SR mirror (SRM) in the output port establishes a carrier light detuned single-sideband SR cavity. Bottom left, extension for a broadband shot-noise reduction utilizing squeezed states. Right, topology, proposed here. Two optically coupled cavities are formed with the help of an additional mirror TSRM. Their resonance doublet enables detuned TSR, resulting in lower shot noise. Squeezed states can be used without an additional filter cavity. 


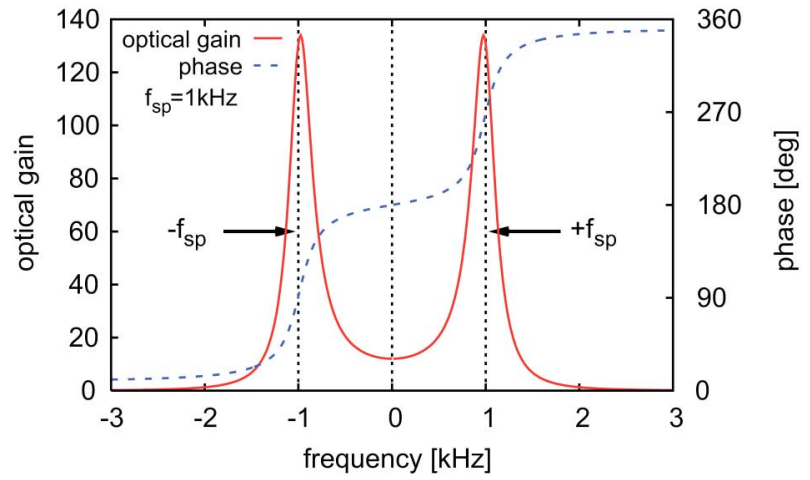

Fig. 2. (Color online) Resonance doublet of two optically coupled Fabry-Perot resonators. The frequency splitting $f_{\mathrm{sp}}= \pm 1 \mathrm{kHz}$ is related to the carrier frequency $f_{0}$, here set to zero.

required for a broadband sensitivity improvement. A table-top prototype of such an interferometer was recently demonstrated. ${ }^{13}$

The right-hand part of Fig. 1 shows the topology proposed here. Starting from the standard SR topology, an additional mirror (TSRM) is placed in the signal output port of the interferometer. This mirror forms a new long-baseline cavity that is optically coupled with the initial SR cavity. Similar to two coupled mechanical oscillators, two coupled optical cavities feature a resonance doublet that has been experimentally observed; see, for example, Ref. 14 . The frequency splitting $\omega_{\mathrm{sp}}$ of this resonance doublet is determined by the coupling of the two resonators, i.e., the transmission of the center mirror (SRM). The transmission $T_{c}$ of the mirror SRM corresponding to this coupling can be obtained by solving the intersection $^{15}$

$$
-\frac{1}{2} \arg \left[\rho_{23}\left(\frac{\omega_{\mathrm{sp}} L_{\mathrm{SR} 2}}{c}\right)\right]=\frac{L_{\mathrm{SR} 1}}{L_{\mathrm{SR} 2}} \frac{\omega_{\mathrm{sp}} L_{\mathrm{SR} 2}}{c},
$$

where $L_{\mathrm{SR} 1}$ is the length of the resonator in the output port (formed by the mirrors SRM and TSRM) and $L_{\mathrm{SR} 2}$ is the length of the resonator formed by the SRM and the interferometers' end mirrors. The term arg $\left(\rho_{23}\right)$ is the frequency-dependent phase of the light field reflected from this resonator determining the resonance condition of the three-mirror cavity. Notice that the macroscopic lengths determine the free spectral ranges of the single resonators. The right-hand side of Eq. (1) describes the dispersion in the two resonators; refer to Ref. 15 for further details. If lengths $L_{\mathrm{SR} 1}$ and $L_{\mathrm{SR} 2}$ are assumed to be equal, the solution of Eq. (1) is given by

$$
T_{c}=1-\frac{4 \cos ^{2}\left(2 \frac{\omega_{\mathrm{sp}} L_{\mathrm{SR} 1}}{c}\right) \rho_{\mathrm{end}}^{2}}{\left(1+\rho_{\mathrm{end}}^{2}\right)^{2}} .
$$

For ideal interferometer end mirrors with reflectivity $\rho_{\text {end }}=1$, Eq. (2) further reduces to

$$
T_{c}=1-\cos ^{2}\left(2 \frac{\omega_{\mathrm{Sp}} L_{\mathrm{SR} 1}}{c}\right) .
$$

Thus the transmission $T_{c}$ of the mirror SRM has to be chosen with respect to the required frequency splitting $\omega_{\mathrm{sp}}=2 \pi f_{\mathrm{sp}}$. Then the resonances' bandwidth of the doublet can be determined by the reflectivity of the mirror TSRM. In this Letter we propose equal lengths $L_{\mathrm{SR} 1}$ and $L_{\mathrm{SR} 2}$ and in the following a frequency splitting $f_{\mathrm{sp}}=1 \mathrm{kHz}$. The reflectivity of TSRM is chosen to give the same peak sensitivity as in the SR case. Figure 2 shows the resonance doublet for $L_{\mathrm{SR} 1}=L_{\mathrm{SR} 2}=1200 \mathrm{~m}, \rho_{\mathrm{MI}}^{2}=0.99995$, and $\rho_{\mathrm{TSRM}}^{2}=0.963$. The resonance doublet is symmetric around the carrier frequency $f_{0}$ in magnitude and phase, respectively. Thus, if the three-mirror coupled cavity is tuned to $f_{0}$, upper and lower signal sidebands have identical resonance conditions.

Figure 3 shows the quantum noise spectral densities of detuned TSR (solid curves) in comparison with detuned SR, using homodyne detection at the signal port. All traces were calculated by using technical design parameters of the GEO 600 detector as used in Ref. 9. In the case of detuned SR the spectral noise density is different for detection of amplitude and phase quadrature. Here we consider detection of the amplitude quadrature that provides low noise spectral densities for frequencies below the optical resonance, here at $1 \mathrm{kHz}$.

Figure 3(a) compares SR and TSR without squeezed field input. The dotted (dotted-dashed)
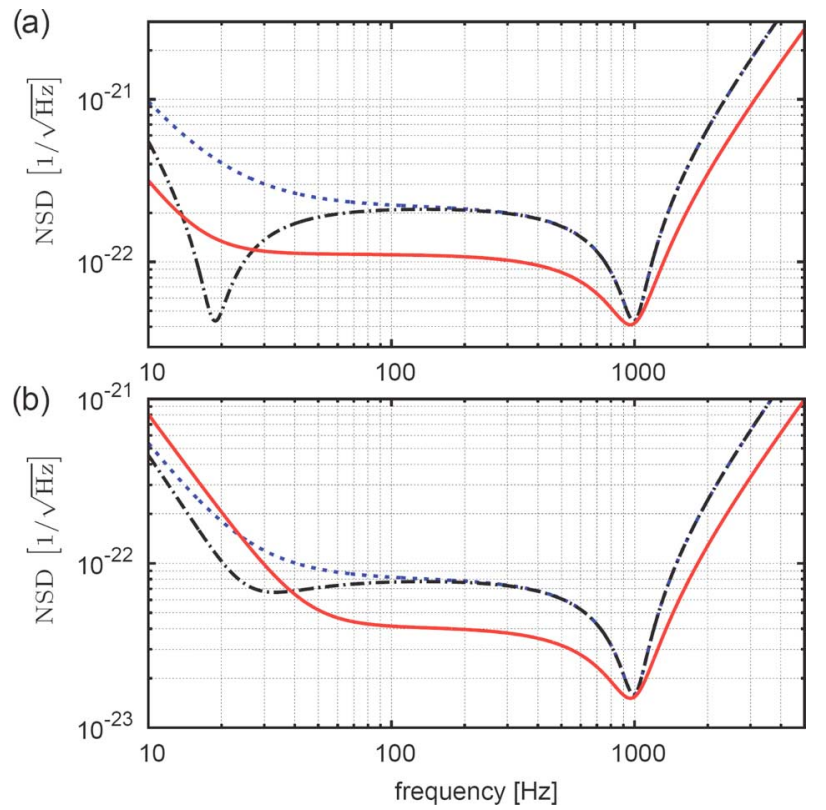

Fig. 3. (Color online) Comparison of linear noise spectral densities (NSD) of TSR (solid curves) with detuned singlesideband SR (a) without squeezed input and (b) with squeezed input $(r=1)$. The dashed-dotted curves represent SR of the upper sideband; the dotted curves represent SR of the lower sideband. In both cases the TSR topology yields a lower noise spectral density, by up to a factor of two, at sideband frequencies above $40 \mathrm{~Hz}$. For a detailed description refer to the text. 
curve corresponds to SR with resonating lower (upper) signal sideband at about $1 \mathrm{kHz}$. For signal frequencies above $40 \mathrm{~Hz}$ the TSR technique proposed here provides an improved noise spectral density. This frequency range corresponds to the shot-noisedominated regime. For lower frequencies, quantum radiation pressure noise dominates shot noise, and the detuned SR topology with resonating lower signal sideband shows a resonance dip at slightly below $20 \mathrm{~Hz}$. This is the so-called optical spring resonance. ${ }^{16}$ Such a resonance does not appear in the TSR topology proposed here, because upper and lower sidebands have detunings symmetrically arranged around the carrier frequency $\omega_{0}$. Note that in the case of TSR, phase modulation as introduced by GWs appears only in the carrier light phase quadrature, as it is expected from a simple Michelson interferometer. This contrasts with detuned SR, where signal sidebands appear in phase and amplitude quadratures and in any linear combination of both.

Figure 3(b) compares the detuned SR with the detuned TSR technique in combination with the injection of squeezed fields, as shown in Fig. 1. Here we assume a broadband squeezed field with squeezing parameter $r=1$, which corresponds to about $8 \mathrm{~dB}$ squeezing of noise variance. Again, for frequencies above $40 \mathrm{~Hz}$, TSR achieves a lower noise spectral density than detuned SR. Comparing TSR with and without squeezed-state input shows that the TSR technique provides quantum noise reduction from squeezed states over a wide band without an additional filter cavity. This advantage is a direct result of the resonance doublet symmetrically arranged around the carrier frequency. Note that for all curves the squeezed states are not optimized for a simultaneous reduction of radiation pressure noise, i.e., a reduction of quantum noise in the frequency band below $40 \mathrm{~Hz}$. Such an optimization is possible for the detuned SR as well as for the detuned TSR case and requires additional filter cavities in both cases. However, this regime is also strongly affected by thermal noise ${ }^{17}$ electronic noise from control loops, and seismic noise. It might therefore turn out that the slightly increased noise spectral density at frequencies below $40 \mathrm{~Hz}$ in TSR will not be significant.

We now consider the application of the TSR technique to $\mathrm{GW}$ detectors. In that case the spectral shape of the quantum noise should be considered with respect to other noise sources. Since all current and planned interferometric GW detectors are designed for room-temperature operation, thermal noise gives a significant contribution to the overall noise floor, ${ }^{17}$ and the resonant sideband frequency of the TSR topology should be chosen and optimized with respect to the detector's thermal noise. Thermal noise arises from thermally driven motion of the suspended mirror test masses and typically falls off toward higher frequencies. Since the rate of detectable GW signals are expected to decrease with increasing frequency, one may design the resonance peak, hav- ing a fixed frequency, fitting the falling thermal noise. If nevertheless one wishes to tune the frequency of the resonance peak, as is possible in the detuned SR technique, mirror TSRM needs to be replaced by a short cavity, for example, in the form of an etalon. The reflectivity of such an etalon and therefore the resonance frequency of the TSR scheme can be changed in situ, for example, by changing the etalon's temperature.

In conclusion, we propose a new interferometer technique aiming at optimum quantum noise reduction at intermediate and upper (shot-noise-limited) detection frequencies of ground-based gravitational wave detectors. The new technique requires a single additional mirror on top of the current GEO 600 topology and solves two problems at the same time. With this technique upper and lower signal sidebands are resonantly enhanced simultaneously, and squeezed states can be used without setting up another long-baseline filter cavity.

A. Thüring's e-mail address is andre.thuering@ aei.mpg.de.

\section{References}

1. D. Sigg (for the LIGO Science Collaboration), Class. Quantum Grav. 23, S51 (2006).

2. M. Ando et al., Phys. Rev. Lett. 86, 3950 (2001).

3. F. Acernese et al., Class. Quantum Grav. 23, S635 (2006).

4. S. Hild (for the LIGO Scientific Collaboration), Class. Quantum Grav. 23, S643 (2006).

5. G. Heinzel, K. A. Strain, J. Mizuno, K. D. Skeldon, B. Willke, W. Winkler, R. Schilling, A. Rüdiger, and K. Danzmann, Phys. Rev. Lett. 81, 5493 (1998).

6. A. Weinstein, Class. Quantum Grav. 19, 1575-1584 (2002).

7. C. M. Caves, Phys. Rev. D 23, 1693 (1981).

8. H. Vahlbruch, S. Chelkowski, B. Hage, A. Franzen, K. Danzmann, and R. Schnabel, Phys. Rev. Lett. 97, 011101 (2006).

9. J. Harms, Y. Chen, S. Chelkowski, A. Franzen, H. Vahlbruch, K. Danzmann, and R. Schnabel, Phys. Rev. D 68, 042001 (2003).

10. H. J. Kimble, Y. Levin, A. B. Matsko, K. S. Thorne, and S. P. Vyatchanin, Phys. Rev. D 65, 022002 (2001).

11. R. Schnabel, J. Harms, K. A. Strain, and K. Danzmann, Class. Quantum Grav. 21, S1045 (2004).

12. This feature has previously been noticed by B. J. Meers and R. W. P. Drever in a draft titled "Doubly resonant signal recycling for interferometric gravitational wave detectors."

13. H. Vahlbruch, S. Chelkowski, B. Hage, A. Franzen, K. Danzmann, and R. Schnabel, Phys. Rev. Lett. 95, 211102 (2005).

14. G. de Vine, M. Gray, D. E. McClelland, Y. Chen, and S. Whitcomb, Phys. Lett. A 316, 17 (2003).

15. A. Thüring, H. Lück, and K. Danzmann, Phys. Rev. E 72, 066615 (2005).

16. A. Buonanno and Y. Chen, Phys. Rev. D 65, 042001 (2002).

17. V. B. Braginsky, M. L. Gorodetsky, and S. P. Vyatchanin, Phys. Lett. A 264, 1 (1999). 